\title{
Dolor orofacial en la clínica odontológica
}

\author{
B. C. Migueláñez Medrán¹, C. Goicoechea Garciaª , A López Sánchez y M. A. Martínez García²
}

'Área de Estomatología. Dpto. Medicina y Cirugía, Psicología, Medicina Preventiva y Salud Pública,
Inmunología y Microbiología Médica, Enfermería y Estomatología. Facultad de Ciencias de la Salud.
Universidad Rey Juan Carlos (URJC). Alcorcón, Madrid. ${ }^{2}$ Área de Farmacología, Nutrición y Bromatología.
Unidad asociada I+D+i al Instituto de Química Médica (CSIC). Grupo de Excelencia investigadora URJC-Banco
de Santander-Grupo Multidisciplinar de Investigación y Tratamiento del Dolor (i+DOL). Dpto. Ciencias Básicas
de la Salud. Facultad de Ciencias de la Salud. Universidad Rey Juan Carlos (URJC). Alcorcón, Madrid. España

\section{ABSTRACT}

Most dental consultations are related to intraoral pain disorders affecting dental, periodontal and mucosal structures. Although the originating cause of pain and the anatomical structure frequently co-localise, orofacial pain and particularly oral pain are sometimes referred. That is, pain may be caused by extraoral processes out of the maxillofacial territory. Likely, some intraoral conditions such as an occlusal imbalance may also affect extraoral structures, leading to tension and pain on the neck, head, and back. Orofacial pain research is however an emerging discipline in comparison to other anatomical regions. This may be due, in part, to the fact that oral pain tends to recede over time or after tissue healing -in case there was an injury-. Notwithstanding, half of the patients reporting any sort of orofacial pain suffers chronically. And unlike acute receding pain, chronic pain is no longer a symptom, but a difficult-to-manage pathology, with scarce or none relation to the mechanisms that caused it. Moreover, the lack of appropriate anamnesis and clinical examinations, inaccurate pain syndrome nomenclatures or difficulty in diagnosis hamper sometimes an optimal therapeutic approach. Most oral pain classifications are still based on the affected anatomical structure rather than on the nociceptive mechanism itself. On the other hand, the precise aetiology of most of the so-called atypical algiae or the burning mouth syndrome is still unknown. The present review article aims to describe the main reasons for pain consultation at the dental clinic, with particular emphasis on the type of pain from a mechanistically point of view: nociceptive, inflammatory, neuropathic, psychogenic or mixed.

Key words: Orofacial pain, neuralgia, odontalgia, oral cancer, temporomandibular joint pain.

\section{RESUMEN}

La mayor parte de las consultas odontológicas están relacionadas con dolores intraorales que afectan a estructuras dentarias, periodontales y mucosas. Aunque generalmente la causa originaria del dolor y la estructura afectada coinciden en la localización, en ocasiones el dolor orofacial y, particularmente, el dolor oral, es referido. Esto es, el dolor puede deberse a procesos de origen extraoral localizados fuera del territorio maxilofacial. De igual manera, determinados trastornos orales, como un desequilibrio oclusivo, pueden afectar también estructuras extraorales, ocasionando tensión y dolor en cuello, cabeza y espalda. La investigación en dolor orofacial es, sin embargo, una disciplina emergente en comparación con otras áreas anatómicas, quizás debido, en parte, a que el dolor tiende a remitir con el tiempo o con la sanación del tejido afectado (si hubiera una lesión). Sin embargo, la mitad de los pacientes con algún tipo de dolor orofacial lo sufre de manera crónica y, a diferencia del dolor agudo, remitente, el dolor crónico no es ya un síntoma, sino una patología de difícil manejo, con escasa o ninguna relación con los mecanismos que lo originaron. Además, la falta de una adecuada anamnesis y exploración clínica, nomenclaturas inapropiadas o la dificultad de diagnóstico, hacen complicado en ocasiones un óptimo abordaje terapéutico. La mayoría de las clasificaciones de dolor oral siguen atendiendo a la estructura anatómica afectada más que al propio mecanismo nociceptivo. Por otra parte, la etiología exacta de muchas algias denominadas atípicas o del síndrome de boca ardiente sigue siendo desconocida. Esta revisión pretende describir los principales motivos de consulta por dolor en la clínica dental, poniendo particular énfasis en el tipo de dolor desde el punto de vista de su mecanismo: nociceptivo, inflamatorio, neuropático, psicogénico o mixto.

Palabras clave: Dolor orofacial, neuralgia, odontalgia, cáncer oral, dolor articular temporomandibular.

Recibido: 16-02-2019

Aceptado: 23-04-2019

Correspondencia: Blanca del Carmen Migueláñez Medrán blancac.miguelanez@urjc.es
Migueláñez Medrán BC, Goicoechea García C, López Sánchez A, Martínez García MA. Dolor orofacial en la clínica odontológica. Rev Soc Esp Dolor 2019;26[4]:233-242. 


\section{INTRODUCCIÓN}

La mayoría de los pacientes que acuden a la clínica dental refieren odontalgias que, por lo general, son de carácter agudo. Sin embargo, el dolor de la musculatura temporomandibular y miofascial, junto a las neuralgias, se encuentran entre los distintos tipos de dolor crónico con una mayor incidencia en la consulta odontológica (1-3). Las cefaleas constituyen otro grupo de gran frecuencia, pero en la población general $(4,5)$. De hecho, de acuerdo con la Sociedad Española del Dolor (SED), la mitad de los pacientes con dolor orofacial en la población general (esto es, sin atender exclusivamente a aquellos pacientes que acuden al odontólogo), lo sufre de manera crónica. Todos estos tipos de dolor son complejos de tratar, siendo más común entre mujeres (a excepción del dental) $(6,7)$ y disminuyendo su prevalencia, por lo general, con la edad (8).

Dada la abundante e intrincada inervación de las regiones asociadas al dolor orofacial (Figura 1), no es de extrañar que, en ocasiones, sea tan difícil de categorizar (9), lo que puede condicionar su abordaje terapéutico y, en consecuencia, la eficacia del tratamiento. Pero, además, la existencia en ocasiones de un dolor de fuerte componente psicogénico, (2) e incluso de tipo referido (10), hace más complicado si cabe dar con un diagnóstico y tratamiento eficaz. Es precisamente la existencia de unos nervios craneales, ajenos a la médula espinal, lo que exige y justifica una estrecha colaboración entre expertos del aparato estomatognático, psicólogos y diversos especialistas médicos, más allá de la existente en las Unidades de Dolor para otras regiones anatómicas. Esto es, se hace necesario el desarrollo de unidades de dolor orofacial $(11,12)$.
Además, el escaso conocimiento sobre ciertos tipos de patologías de dolor orofacial por parte de diversos profesionales sanitarios no es un hecho raro, como se recoge en trabajos monográficos realizados por el Colegio de Odontólogos y Estomatólogos de España (13). Por todo ello, la presente revisión tiene por objeto describir y clasificar los principales motivos de consulta que pueda encontrar el odontólogo en su práctica diaria, con la aparición de un proceso doloroso como desencadenante de la visita odontológica como denominador común.

\section{PRINCIPALES TIPOS DE DOLOR OROFACIAL}

La mayor parte de los pacientes que experimentan algún tipo de dolor orofacial acuden a su médico de cabecera u odontólogo y, habitualmente, son tratados por ellos mismos. Sin embargo, en algunas ocasiones el paciente debe ser remitido a un especialista 0 , incluso, a una unidad de dolor.

En línea con lo comentado anteriormente, aunque un elevado porcentaje de algias tienen su origen en estructuras dentarias, periodontales y mucosas, existen determinadas afecciones que pueden encontrar procesos dolorosos en estas mismas estructuras derivados de otras localizaciones extraorales (10). Una de las características a tener en cuenta en el diagnóstico del dolor orofacial (y más concretamente en el dolor oral) es el hecho de que las algias pueden tener un origen diverso (dentario, oral o incluso sistémico], influenciado además por otras sensaciones subjetivas del propio paciente, como conductas depresivas o la ansiedad (14). Llegar a un correcto diagnóstico resulta en ocasiones complicado, ya que muchos tipos de dolor, aun con mecanismos de nocicepción diferentes, comparten signos y síntomas $(15,16)$.

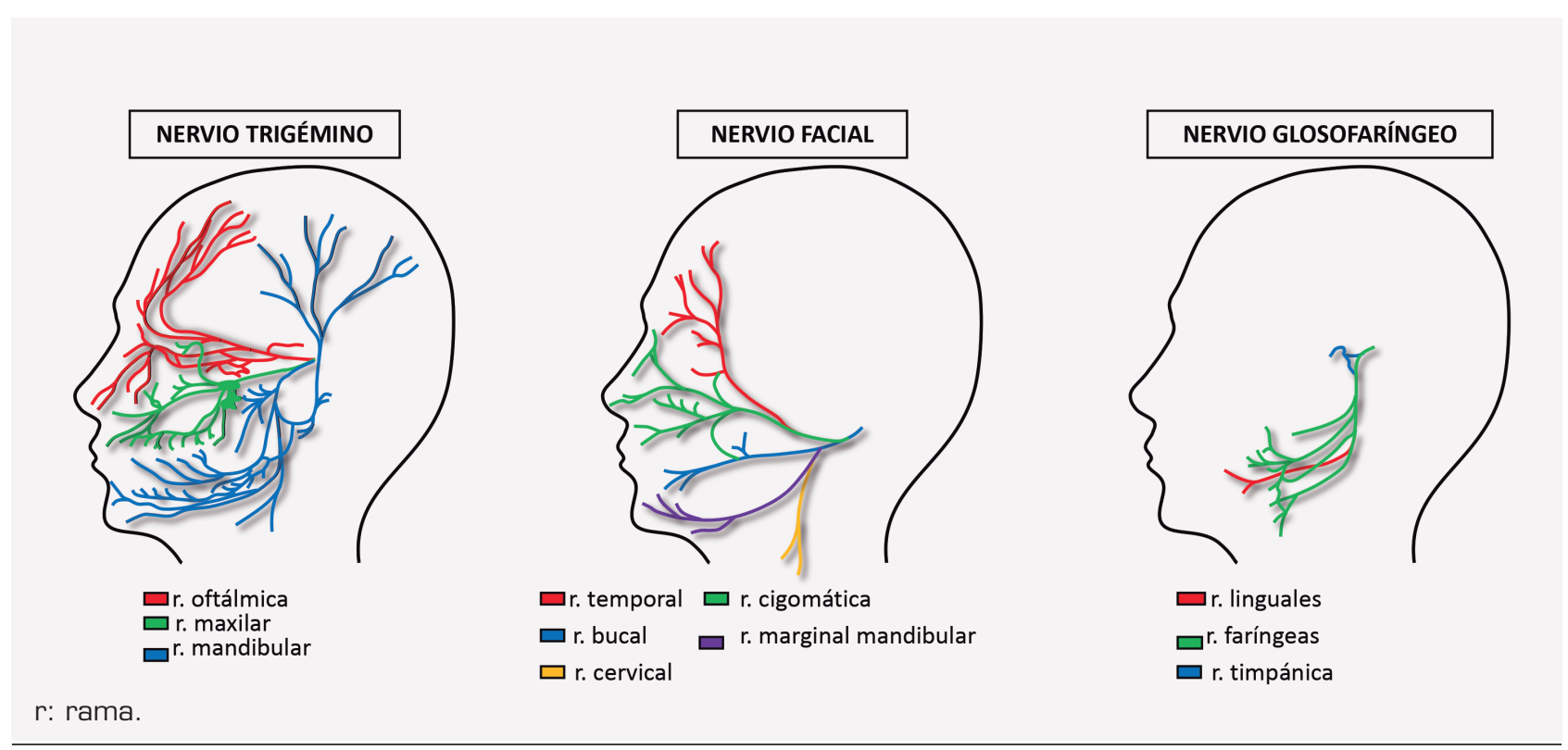

Fig. 1. Representación esquemática de los nervios trigémino, facial y glosofaríngeo. Las vías tanto motoras como sensitivas se muestran indistintamente representadas. 


\section{Odontalgias}

La pulpa dental está densamente inervada por nociceptores $C$ polimodales, pero también por fibras $A \delta$ y $A B$, lo que permite responder a estímulos de diverso origen. Se piensa que, en la mayoría de los casos, el dolor dentario es consecuencia de un proceso inflamatorio de la pulpa y su duración e intensidad depende generalmente de la magnitud del daño, cediendo al remitir el estímulo que lo produce. El principal motivo de hipersensibilidad dental es, por tanto, la exposición de los túbulos dentinarios a estímulos térmicos o mecánicos, a la ingesta de alimentos dulces o a la presión ejercida por el cepillado (17). La causa desencadenante suele ser la exposición de la dentina a procesos de atrición, abrasión o erosión (como la producida por las caries [18,19]), aunque también puede deberse a la exposición de la superficie radicular del diente de manera secundaria a una enfermedad periodontal o derivada de una intervención quirúrgica (20). En contraposición a esto, se ha propuesto la existencia de algoneuronas: fibras $A \beta$ mecánicas de bajo umbral que transmitirian señales nociceptivas en ausencia de inflamación ni sensibilización central al ser estimuladas mecánicamente (por ejemplo, por un soplo de aire o un chorro de agua); esto es, estarían activas de manera constitutiva en el diente sano y quedarían expuestas al erosionarse el esmalte y la dentina (21).

La fractura de un diente puede dar lugar también a un proceso doloroso conocido como sindrome del diente fisurado. La dificultad en el manejo de este tipo de dolor estriba en que la detección de la fractura y su profundidad son difíciles de valorar, ya que no existe una pérdida estructural o separación visible de la estructura dentaria (22). Los métodos utilizados para su visualización en la clínica odontológica consisten en la transiluminación, en técnicas radiográficas o en la aplicación de azul de metileno $(23,24)$. Sin embargo, no siempre es posible ver dicha fractura (25), lo que dificulta un diagnóstico y tratamiento adecuado. Su tratamiento depende de la profundidad de la fisura y del tejido afectado: esmalte, dentina, cavidad pulpar y/o superficie radicular (22).

En ocasiones, infecciones o lesiones de las piezas dentales posteriores, elevaciones de seno incorrectamente realizadas, sobreobturaciones de conductos radiculares en endodoncias, complicaciones derivadas de la colocación de implantes dentales e incluso fístulas oroantrales resultantes de una extracción dentaria pueden dañar la membrana bucosinusal, dando lugar a una sinusitis maxilar de origen odontogénico (26-30). La principal consecuencia es una percepción de mal olor aproximadamente por la mitad de los propios pacientes y un aumento de la susceptibilidad a infecciones microbianas. Aunque estudios previos afirman que solo un $\sim 30 \%$ de estos pacientes presenta dolor dental (31), no están exentos de tratamiento o extracción de la pieza afectada, tratamiento antibiótico, así como de ser derivados al servicio de cirugía maxilofacial $(29,32)$.

\section{Dolor somático superficial: mucosa y periodonto}

La estomatitis aftosa recidivante es la enfermedad más común de la mucosa oral. Cursa con ulceraciones recurrentes que provocan dolor, persistiendo durante días o semanas $(33,34)$. Su etiología aún es desconocida y el tratamiento actual es sintomático y encaminado a disminuir el número y tamaño de las ulceraciones (35). Existe también un grupo de enfermedades autoinmunes que afecta a la piel y mucosa oral, cursando con dolor de tipo inflamatorio. Dos de estas enfermedades son el pénfigo, en la cual se producen ampollas en la superficie cutánea y mucosa, y el penfigoide, de afectación casi exclusivamente mucosa, que puede afectar desde la mucosa oral a la nasal, ocular o incluso genital (36). El liquen plano oral constituye otra enfermedad de carácter autoinmune particular. Se manifiesta en forma de lesiones blanquecinas de la mucosa que producen dolor, ardor y escozor (37). En ambos casos, el tratamiento consiste en la aplicación local de corticosteroides; también se puede optar en casos más severos por el empleo de retinoides, inmunomoduladores o fototerapia para lesiones cutáneas, así como el uso de antisépticos y analgésicos para controlar el dolor. Las principales complicaciones en el tratamiento de este tipo de patologías residen en su carácter recidivante y en el riesgo potencial de desarrollar micosis al ser pacientes sometidos a un tratamiento de larga duración con corticoides (38).

Existen también distintos tipos de dolor periodontal. Una de las principales causas de dolor de origen periodontal viene dado por trauma oclusal, en el cual las fuerzas oclusales sobre el periodonto exceden la capacidad adaptativa de los tejidos. Si ese aumento de las fuerzas oclusales se mantiene en el tiempo, además puede dar lugar a patología de tipo articular. Su tratamiento incluye analgésicos y la realización de ajustes oclusales para disminuir la fuerza aplicada a la pieza dental afectada, corrección y manejo de hábitos parafuncionales, ferulización de las piezas con movilidad, tratamiento ortodóncico, reconstrucción oclusal con diferentes tratamientos protéticos o en último caso extracción de la pieza implicada (39). Otras dos formas específicas de enfermedad periodontal son la gingivitis ulcerativa necrotizante y la periodontitis ulcerativa necrotizante. Ambas se caracterizan por un proceso agudo de fuerte dolor gingival, necrosis de las papilas y sangrado $(40,41)$, con la diferencia de que la segunda cursa además con afectación ósea (42). Además del tratamiento sintomático con analgésicos, la terapia farmacológica incluye el uso de terapia antibiótica que combine amoxicilina y metronidazol y enjuagues antibacterianos (clorhexidina) o antisépticos (peróxido de hidrógeno); además se hace necesario el uso de terapia mecánica para eliminar la placa bacteriana (43).

Diversas afecciones de las glándulas salivales pueden cursar también con dolor. La sialometaplasia necrotizante es un proceso inflamatorio que genera una superficie ulcerada, dolorosa o no, en las glándulas salivales del paladar duro (44-46). Su aparición se asocia mayoritariamente a la aplicación de anestesia en el paladar duro y al efecto vasoconstrictor que esta produce (47). Por el contrario, aunque también de carácter inflamatorio, la sialoadenitis necrotizante aguda es un proceso de etiología desconocida que afecta principalmente a las glándulas salivales menores y se caracteriza por la aparición de un fuerte dolor en paladar duro, blando o amígdalas (tonsilas) de una duración aproximada entre 
5-6 semanas. Otra afección de las glándulas salivales es la sialolitiasis, la cual cursa con la formación de cálculos salivales (sialolitos) en el parénquima del conducto de una glándula salival (48). La oclusión del conducto impide el paso de saliva y se produce un aumento de la presión intraductal, responsable de la aparición de la sensación dolorosa y la tumefacción (49); no obstante, en algunos casos no se experimenta dolor (50). En este caso, se pueden administrar alimentos o incuso fármacos (sialogogos) que estimulen la secreción salival y que, de esta forma, se produzca la expulsión del sialolito. Además, el tratamiento puede precisar de terapia antibiótica para evitar un proceso infeccioso, drenaje de la glándula, extirpación del sialolito (51) o incluso fragmentación mediante ultrasonidos (52).

Diversas infecciones bacterianas, micóticas y víricas de la cavidad oral pueden cursar con dolor, siendo el tratamiento para todas ellas exclusivamente sintomático, con analgésicos y antibióticos, antifúngicos o antivirales. Las inflamaciones tanto agudas como crónicas pueden afectar a las glándulas salivales mayores (parótida, submaxilar y submandibular] y en menor grado a las menores también (53). La glándula parótida es la más afectada por estos procesos infecciosos $(54,55)$. A tal efecto, la parotiditis aguda bacteriana produce una tumefacción inflamatoria que se caracteriza por la aparición de dolor intenso, fiebre y malestar general. Los mismos síntomas son apreciados en la parotiditis aguda epidémica (paperas) de origen vírico (56). Por su parte, el contagio del virus herpes simple se produce por contacto de un individuo sano con otro infectado con lesiones activas cutáneas o de la mucosa oral o incluso por fómites. Una vez producida la primoinfección herpética, el virus permanece latente y su reactivación podrá dar lugar a herpes labiales o intraorales, con la aparición de múltiples vesículas que se unirán formando úlceras más grandes. Estas ulceraciones se caracterizan por sensaciones urentes, de hormigueo y dolor (57). Entre las infecciones micóticas, la candidiasis es la más frecuente en la cavidad oral. La candidiasis oral cursa con lesiones blanquecinas o eritematosas localizadas en lengua, mucosa yugal, paladar, rebordes alveolares, amígdalas e incluso esófago $(58,59)$ y, en ocasiones, dichas lesiones pueden causar odinofagia y disfagia.

A pesar del carácter recurrente de la mayoría de estas afecciones, todas implican un tipo de dolor agudo puntual, solventable o al menos capaz de ser reducido en gran medida con los analgésicos comúnmente pautados.

\section{Síndrome de boca ardiente}

El síndrome de boca ardiente constituye un capítulo aparte dentro de los distintos tipos de dolor orofacial. Su principal característica es la aparición de una sensación dolorosa de ardor o escozor en la parte anterior de la lengua, aunque pueden verse afectadas otras localizaciones tales como el paladar, rebordes alveolares, mucosa yugal y labios (60). Presenta una duración de, al menos, 4 o 6 meses, sin que puedan objetivarse lesiones físicas, es decir, a la exploración, la cavidad oral presenta un aspecto sin ninguna patología objetivable. Afecta principalmente a mujeres en edad postmenopáusica [61) y su prevalencia se ha estimado en un $0,7-4,6 \%$ de la población general (62). Es una enfermedad de curso crónico y, aunque se sospecha la implicación de componentes neuropáticos y psicogénicos, la terapia empleada sigue siendo poco efectiva y complicada de manejar (63-65). Actualmente, el tratamiento consiste en un abordaje multidisciplinar, con la aplicación tópica de capsaicina, benzodiacepinas y corticoides, así como el uso de terapia psicológica (66).

\section{Dolor somático profundo: dolor muscular y articular}

Las afecciones funcionales del sistema estomatognático afectan en torno a un $80 \%$ de la población mundial (67) y, a tal efecto, los trastornos temporomandibulares constituyen una de las causas más frecuentes de dolor crónico orofacial (68), afectando tanto a la articulación temporomandibular como a los músculos masticatorios y estructuras adyacentes (69). Otra de las afecciones más frecuentes en la población general, y por tanto de visita a los centros de atención primaria (70,71), viene representada por el dolor miofascial. Aunque se caracteriza por la aparición de puntos gatillo sobre músculos faciales, su etiología aún no es del todo conocida y, en ausencia de un tratamiento específico (72), actualmente se opta por un manejo multiterapéutico: terapia física manual, electroterapia, láser de baja intensidad, terapia con ultrasonidos, punción seca, fármacos antinflamatorios no esteroideos (AINE), parches de lidocaína, así como relajantes musculares o benzodiacepinas (71). La elevada presencia de estos tipos de dolor en la sociedad, sumado a su difícil manejo terapéutico, hacen necesario un mayor estudio sobre su etiología, fisiopatología y tratamiento en general.

\section{Dolor neuropático}

Las neuralgias constituyen un tipo de dolor orofacial con una prevalencia difícil de calcular, en muchos casos, debido a su etiología desconocida. Aunque el estudio de las neuralgias orofaciales se ha limitado tradicionalmente al nervio trigémino, recientemente se están abriendo nuevas clasificaciones para el estudio de diferentes neuralgias: neuralgia del trigémino, dolor neuropático trigeminal atípico, dolor facial idiopático persistente (dividido en dolor facial atípico y odontalgia atípica], neuralgia del nervio intermedio de Wrisberg (o geniculado), neuralgia del nervio glosofaríngeo, neuralgia del nervio laríngeo superior, neuralgia postherpética, dolor neurovascular atípico, dolor dental fantasma, etc. $(73,74)$.

La neuralgia del trigémino representa uno de los dolores orofaciales más complejos de tratar. El daño nervioso puede presentar una localización y etiología diversa, habiéndose desarrollado hasta el momento una clasificación en clave dicotómica: neuralgia clásica, producida por la compresión microvascular en la entrada del nervio al tallo cerebral, y sintomática, todas las demás (75). La sensación dolorosa puede durar desde unos segundos a varios minutos $y$, aunque muchos pacientes presentan picos de dolor, este suele estar presente en mayor o menor medida de manera 
constante (76). Su prevalencia aún no es del todo bien conocida $(77,78)$ y el tratamiento farmacológico corresponde mayoritariamente al uso de anticonvulsivantes/ antiepilépticos, aunque también se prescriben antipsicóticos y benzodiacepinas (76). Algunos pacientes, no obstante, parecen ser refractarios al tratamiento farmacológico, existiendo actualmente para ellos otras opciones terapéuticas: el tratamiento quirúrgico por medio de técnicas percutáneas del ganglio de Gasser o por descompresión microvascular $[79,80)$.

Aunque el dolor facial idiopático persistente ha sido tradicionalmente clasificado como un dolor somático que afecta a estructuras musculares dado su carácter sordo y difícilmente localizable (en contraposición a la neuralgia del trigémino, caracterizada por un dolor punzante e intenso] (65), la persistencia de este tipo de dolor a lo largo del tiempo y su difícil diagnóstico hacen sospechar una afectación neuropática $(76,79)$. A pesar de que en la mayoría de las ocasiones el dolor dental de los pacientes que acuden a la clínica odontológica corresponde a un proceso oral identificable y, por tanto, el tratamiento se realiza acorde a la etiología del proceso que causa el dolor (81), en ocasiones encontramos odontalgias atipicas que parecen también implicar un componente mixto o neuropático más difícil de tratar (82). Este dolor puede originarse tanto en la complicación de la lesión de una pieza dental como de manera subsecuente a su extracción (síndrome del diente fantasma), pero sin ningún signo clínico ni radiográfico que evidencie patología existente [83]. Además de su carácter neuropático, las odontalgias atípicas (y neuralgias orofaciales en su conjunto] generalmente implican un fuerte componente psicógeno (84); por ello, su tratamiento farmacológico incluye la prescripción de antidepresivos y/o antipsicóticos, benzodiacepinas o antiepilépticos (85-88).

Mención especial merece el dolor mandibular de origen cardiaco. La isquemia aguda de miocardio cursa generalmente con dolor retroesternal que puede proyectarse hacia brazos, cuello y mandíbula. No obstante, en determinadas ocasiones el dolor queda confinado al territorio maxilofacial, frecuentemente en cuello y mandíbula, aunque también lo encontramos en forma de dolor intraoral. Este último podría explicarse por las conexiones interneuronales existentes entre los niveles medulares del nervio trigémino y las raíces cervicales superiores (10).

\section{Dolor oncológico}

El carcinoma de células escamosas (o carcinoma epidermoide) es el tumor maligno más frecuente en la cavidad oral ( $90 \%$ de los tumores malignos encontrados en la región oral) $(89,90)$. Se caracteriza por un crecimiento invasivo, una tasa muy elevada de recidivas tempranas y frecuentes metástasis en los nódulos linfáticos cervicales (91). Cursa, en ocasiones, con dolor en estadios avanzados, siendo asintomático en estadios tempranos, inflamación y cambios en la mucosa oral (92). Debido a que los pacientes con un estadio avanzado de carcinoma oral tienen un mal pronóstico a medio-largo plazo (93), el diagnóstico temprano y el reconocimiento de ciertas lesiones precancerosas resultan de vital importancia (94).
De manera adicional, el tratamiento radio/quimioterápico en pacientes oncológicos no está exento de complicaciones orales, independientemente de la localización del tumor. A este respecto, la mucositis constituye una consecuencia del tratamiento oncológico que aparece en localizaciones intraorales recubiertas por mucosa no queratinizada (mucosa labial y yugal, superficies ventral y lateral de la lengua, paladar blando y suelo de la boca) (95). Además de cursar con dolor, la mucositis oral genera una mayor probabilidad de infecciones, dificulta la ingesta y, por ende, la tasa de comorbilidad en este tipo de pacientes es elevada (96). En la actualidad, el tratamiento es eminentemente sintomático, concomitante al tratamiento oncológico.

\section{Cefaleas}

El manejo de las cefaleas experimentó una evidente mejoría con la creación en 1988 de la Clasificación Internacional del Dolor de cabeza (International Headache Classification), con la cual, no solo el diagnóstico, sino también el conocimiento de las frecuencias de los distintos tipos de dolor en la sociedad y sus respectivos tratamientos han evolucionado ampliamente (97). Aunque la base fisiopatológica de los diferentes tipos de cefaleas puede ser muy diversa, en todos ellos existe una sensibilización de las aferencias del nervio trigémino (a nivel intra o extracraneal) (98).

De entre los distintos tipos cefaleas, el dolor de cabeza tensional constituye la cefalea más común (97), con una prevalencia en torno al $40 \%$ (99). Sin embargo, su levemoderada intensidad y su difícil diagnóstico han favorecido que sea infratratado con respecto a otros tipos de cefaleas de dolores más intensos y localizados. De hecho, hoy en día el tratamiento de la cefalea tensional por lo general no es farmacológico, sino que responde preferentemente a técnicas de fisioterapia. Aunque el mecanismo patofisiológico concreto no se conoce con exactitud, un aumento de la sensibilidad y de la dureza de los tejidos pericraneales y miofasciales parece preceder a este tipo de dolor (100), habiéndose sugerido también la participación de factores psicogénicos como otra posible causa, aunque aún está por concretarse si de manera alternativa o complementaria. Por su parte, el dolor de cabeza recurrente ha sido identificado como un trastorno neurológico también de elevada prevalencia en la población general (101).

En determinados casos, hay factores que hacen predecir la aparición del dolor de cabeza, como en el caso de las migrañas o la cefalea en racimos, que pueden precederse de un aura migrañosa previa. El tratamiento de estos tipos de dolor depende en gran medida del agente etiológico desencadenante. En la mayoría de los casos se opta por la administración de antinflamatorios no esteroideos (AINE) u opioides; sin embargo, debido quizás al desconocimiento de la fisiopatología de estos procesos, el alivio del dolor no puede considerarse en muchos casos adecuado (102).

Otros estudios han sugerido que la administración combinada de ácido acetilsalicílico, paracetamol y cafeína resulta más efectiva que el consumo de cada componente de forma aislada o incluso que la combinación de únicamente dos de ellos (103). En cambio, en cefaleas posteriores a la extirpación de un tumor craneal ha sido probada la eficacia del verapamilo y divalproato sódico (104). No obstante, 
la falta de modelos animales apropiados para el estudio de estos tipos de dolor podría explicar en parte el escaso conocimiento sobre su fisiopatología y el tratamiento refractario o ineficaz en muchos de estos pacientes.
A modo de resumen, se recogen las principales dolencias orofaciales observadas en la práctica odontológica y el tipo de dolor atendiendo puramente al mecanismo de acción en la Tabla I.

TABLA I

PRINCIPALES DOLENCIAS OROFACIALES OBSERVADAS EN LA PRÁCTICA ODONTOLÓGICA Y EL TIPO DE DOLOR ATENDIENDO A SU MECANISTICA. LA CLASIFICACIÓN DE LAS CEFALEAS ATIENDE A LOS CRITERIOS ESTIPULADOS POR LA INTERNATIONAL HEADACHE SOCIETY: PRIMER NIVEL DE LA CLASIFICACIÓN INTERNACIONAL DE DOLORES DE CABEZA (75)

\begin{tabular}{|c|c|c|c|c|c|c|}
\hline \multicolumn{4}{|c|}{ Odontalqia } & \multicolumn{3}{|c|}{ Síndrome de boca ardiente } \\
\hline \multirow{3}{*}{\multicolumn{4}{|c|}{$\begin{array}{l}\text { Hipersensibilidad producida por caries } \\
\text { Hipersensibilidad secundaria a enfermedad } \\
\text { periodontal } \\
\text { Hipersensibilidad derivada de una intervención } \\
\text { quirúrgica } \\
\text { Síndrome de diente fisurado } \\
\text { Sinusitis maxilar de origen odontogénico }\end{array}$}} & \multicolumn{3}{|c|}{ Dolor oncológico } \\
\hline & & & & \multirow{2}{*}{\multicolumn{2}{|c|}{$\begin{array}{l}\begin{array}{l}\text { Secundaria al tratamiento radio/ } \\
\text { quimioterápico }\end{array} \\
\begin{array}{l}\text { Carcinoma epidermoide de células } \\
\text { escamosas }\end{array}\end{array}$}} & Mucositis oral \\
\hline & & & & & & $\begin{array}{l}\text { Lengua } \\
\text { Mucosa oral } \\
\text { Hueso mandibular }\end{array}$ \\
\hline \multicolumn{4}{|c|}{ Dolor somático superficial } & \multicolumn{3}{|c|}{ Otros tipos de cáncer } \\
\hline \multirow{2}{*}{\multicolumn{2}{|c|}{ Mucosa }} & \multirow{2}{*}{\multicolumn{2}{|c|}{$\begin{array}{l}\text { Estomatitis aftosa recidivante } \\
\text { Pénfigo } \\
\text { Líquen plano oral }\end{array}$}} & \multicolumn{3}{|c|}{ Cefaleas } \\
\hline & & & & \multirow{2}{*}{ Primarias } & \multirow{2}{*}{\multicolumn{2}{|c|}{$\begin{array}{l}\text { Migraña } \\
\text { Cefalea tensional } \\
\text { Cefalea en racimos y otras cefaleas } \\
\text { autonómicas trigeminales } \\
\text { Otras cefaleas primarias }\end{array}$}} \\
\hline \multicolumn{2}{|l|}{ Periodonto } & \multicolumn{2}{|c|}{$\begin{array}{l}\text { Trauma oclusal } \\
\text { Gingivitis ulcerativa necrotizante } \\
\text { Periodontitis ulcerativa necrotizante }\end{array}$} & & & \\
\hline \multicolumn{2}{|c|}{$\begin{array}{l}\text { Mucosa y/o } \\
\text { periodonto }\end{array}$} & \multicolumn{2}{|c|}{$\begin{array}{l}\text { Virus herpes simple } \\
\text { Candidiasis }\end{array}$} & \multirow{5}{*}{ Secundarias } & \multirow{5}{*}{\multicolumn{2}{|c|}{$\begin{array}{l}\text { Dolor de cabeza atribuible a trauma en } \\
\text { cuello o cráneo } \\
\text { Dolor de cabeza atribuible a un trastorno } \\
\text { vascular cervical o craneal } \\
\text { Dolor de cabeza atribuible a un trastorno } \\
\text { intracraneal no vascular } \\
\text { Dolor de cabeza atribuible a alguna } \\
\text { sustancia o a su retirada } \\
\text { Dolor de cabeza atribuible a un proceso } \\
\text { infeccioso } \\
\text { Dolor de cabeza atribuible a un trastorno } \\
\text { en la homeostasis } \\
\text { Dolor de cabeza o dolor facial atribuible } \\
\text { a un trastorno del cráneo, cuello, } \\
\text { ojos, orejas, nariz, senos paranasales, } \\
\text { dientes, boca u otras estructuras } \\
\text { faciales o craneales } \\
\text { Dolor de cabeza atribuible a un trastorno } \\
\text { psiquiátrico }\end{array}$}} \\
\hline $\begin{array}{l}\text { Glándulas } \\
\text { salivales }\end{array}$ & & $\begin{array}{l}\text { Sialome } \\
\text { Siaload } \\
\text { Sialolitic } \\
\text { Parotidi } \\
\text { Parotidi } \\
\text { (papera }\end{array}$ & $\begin{array}{l}\text { asia necrotizante } \\
\text { s necrotizante aguda } \\
\text { guda bacteriana } \\
\text { guda epidémica }\end{array}$ & & & \\
\hline \multicolumn{4}{|c|}{ Dolor somático profundo } & & & \\
\hline \multicolumn{4}{|c|}{$\begin{array}{l}\text { Miofascial } \\
\text { Musculatura masticatoria y temporal } \\
\text { Músculos del cuello y espalda } \\
\text { [origen oclusal] }\end{array}$} & & & \\
\hline Articular & & $\begin{array}{l}\text { stornos } \\
\text { iporoma }\end{array}$ & $\begin{array}{l}\text { a articulación } \\
\text { ular }\end{array}$ & & & \\
\hline \multicolumn{7}{|c|}{ Neuralgia } \\
\hline \multicolumn{4}{|c|}{ Neuralgia del trigémino } & \multicolumn{3}{|c|}{ Neuralgia postherpética } \\
\hline \multicolumn{4}{|c|}{ Dolor trigeminal atípico } & \multicolumn{3}{|c|}{ Dolor neurovascular atípico } \\
\hline \multicolumn{3}{|c|}{$\begin{array}{l}\text { Dolor facial idiopático } \\
\text { persistente }\end{array}$} & $\begin{array}{l}\text { Dolor facial atípico } \\
\text { Odontalgia atípica }\end{array}$ & \multicolumn{3}{|c|}{ Síndrome del diente fantasma } \\
\hline \multicolumn{4}{|c|}{$\begin{array}{l}\text { Neuralgia del nervio intermedio de Wrisberg [o } \\
\text { geniculado] }\end{array}$} & \multicolumn{3}{|c|}{ Dolor mandibular de origen cardiaco } \\
\hline \multicolumn{4}{|c|}{ Neuralgia del nervio glosofaríngeo } & \multicolumn{3}{|c|}{$\begin{array}{l}\text { Neuralgias craneales, dolor facial central y primario y } \\
\text { otras cefaleas }\end{array}$} \\
\hline \multicolumn{4}{|c|}{ Neuralgia del nervio laríngeo superior } & \multicolumn{3}{|c|}{ Neuralgias craneales y causas centrales de dolor facial } \\
\hline
\end{tabular}




\section{CONCLUSIONES}

A pesar de la existencia de clasificaciones para distintos tipos de dolor orofacial, las revisiones basadas en la evidencia clínica hacen visible la falta de una nomenclatura y una metodología común. Esto complica no solo el diagnóstico, sino también el estudio y abordaje terapéutico de los distintos tipos de dolor orofacial. Los trabajos basados en encuestas o historiales clínicos atienden muchas veces de manera exclusiva a vocablos descriptivos de la sensación experimentada (por ejemplo, urente, lancinante, irruptivo o pulsátil), a acciones (por ejemplo, masticar, comer o abrir la boca) o a localizaciones anatómicas (en ocasiones de manera muy general [por ejemplo, oído, alrededor del ojo, cabeza u otras regiones[], sin entrar a valorar el tipo de dolor de acuerdo a su mecanismo.

En la práctica clínica, el uso de muchos fármacos analgésicos está condicionado por la duración e intensidad del dolor lasí se contempló hace 30 años en la escalera analgésica de la OMS para el manejo del dolor oncológico y se ha venido aplicando a cualquier tipo de dolor), cuando debería depender del mecanismo fisiopatológico propio del tipo de dolor. De hecho, los opioides, frecuentemente indicados para tratar el dolor moderado-severo, fracasan en tratar algunos tipos de dolor crónico.

\section{CONFLICTO DE INTERESES}

Los autores declaran no tener conflicto de intereses.

\section{BIBLIOGRAFÍA}

1. Wirz S, Ellerkmann RK, Buechaler M, Putensen C, Nadstawek J, Wartenberg HC. Management of chronic orofacial pain: a survey of general dentists in German university hospitals. Pain Medicine 2010;11(3):416-24. DOI: 10.1111/j.1526-4637.2010.00805.x

2. Tomoyasu Y, Higuchi H, Mori M, Takaya K, Honda Y, Yamane A, et al. Chronic orofacial pain in dental patients: retrospective investigation over 12 years. Acta Med Okayama. 2014;68(5):269-75. DOI: 10.18926/AMO/52895.

3. Horst OV, Cunha-Cruz J, Zhou L, Manning W, Mancl L, DeRouen TA. Prevalence of pain in the orofacial regions in patients visiting general dentists in the Northwest Practicebased REsearch Collaborative in Evidence-based DENTistry research network. J Am Dent Assoc. 2015;146(10):721-8. e3. DOI: 10.1016/j.adaj.2015.04.001.

4. Koopman JS, Dieleman JP, Huygen FJ, de Mos M, Martin CG, Sturkenboom MC. Incidence of facial pain in the general population. Pain. 2009;147(1-3):122-7. DOI: 10.1016/i. pain.2009.08.023.

5. De Siqueira SRDT, Vilela TT, Florindo AA. Prevalence of headache and orofacial pain in adults and elders in a Brazilian community: an epidemiological study. Gerodontology. 2015;32(2):123-31. DOI: 10.1111/ger.12063.

6. Bassols A, Bosch F, Campillo M, Cañellas M, Baños JE. An epidemiological comparison of pain complaints in the general population of Catalonia (Spain). Pain 1999;83(1):9-16.

7. Riley JL 3rd, Gilbert GH, Heft MW. Orofacial pain symptom prevalence: selective sex differences in the elderly? Pain 1998;76(1-2):97-104.
8. Willeman Bastos Tesch LV, de Souza Tesch R, Pereira Jr FJ. Trastornos temporomandibulares y dolor orofacial crónico: al final, ¿a qué área pertenecen? Rev Soc Esp Dolor. 2014;21(2):70-4. DOI: 10.4321/S1134 80462014000200002

9. Fernández Fernández C. Guía para el abordaje del dolor orofacial. Madrid: Enfoque Editorial SC (Copyright: Grünenthal Pharma S.A.); 2016. p. 1-46.

10. Sáez Yuguero MR, Bermejo Fenoll A, Calvo Guirado JL, Álvarez Martínez E. Dolor mandibular de origen cardiaco. Av Odontoestomatol. 2003;19(5):219-23.

11. Murray H, Locker D, Mock D, Tenenbaum HC. Pain and the quality of life in patients referred to a craniofacial pain unit. J Orofac Pain. 1996;10(4):316-23.

12. Wolf E, Birgerstam P, Nilner M, Petersson K. Patients' experiences of consultations for nonspecific chronic orofacial pain: A phenomenological study. J Orofac Pain. 2006;20(3):226-33

13. de la Hoz Aizpúrua JL. Actualización en disfunción craneomandibular y dolor orofacial. RCOE. 2013;18(3):157-9.

14. Bender SD. Orofacial pain and headache: a review and look at the commonalities. Curr Pain Headache Rep. 2014;18(3):400. DOI: 10.1007/s11916-013-0400-5.

15. Koopman JS, Dieleman JP, Huygen FJ, de Mos M, Martin CG, Sturkenboom MC. Incidence of facial pain in the general population. Pain. 2009;147(1-3):122-7. DOI: 10.1016/j. pain.2009.08.023.

16. Madlan G, Feinmann C. Chronic facial pain: a multidisciplinary problem. J Neurol Neurosurg Psychiatry. 2001;71(6):7169. DOI: 10.1136/jnnp.71.6.716.

17. Bender IB. Pulpal pain diagnosis. A review. J Endod. 2000;26(3):175-9. Dol: 10.1097/00004770$200003000-00012$

18. Tjäderhane L, Larjava H, Sorsa T, Uitto VJ, Larmas $\mathrm{M}$, Salo T. The activation and function of host matrix metalloproteinases in dentin matrix breakdown in caries lesions. J Dent Res. 1998;77(8):1622-9. DOI: 10.1177/00220345980770081001.

19. Han CL, Liewehr FR. Relationships between caries bacteria, host responses and clinical signs and symptoms of pulpitis. Pathogenesis of Pulpitis 2007;33(3):213-9. DOI: 10.1016/j.joen.2006.11.008.

20. Minkoff S, Axelrod S. Efficacy of strontium chloride in dental hypersensivity. J Periodontol. 1987;58(7):470-4. DOl: 10.1902/jop.1987.58.7.470.

21. Fried K, Sessle BJ, Devor M. The paradox of pain from the tooth-pulp: low-threshold "algoneurons"? Pain. 2011;152(12):2685-9. DOI: 10.1016/j. pain.2011.08.004.

22. Ellis SG. Incomplete tooth fracture. Proposal for a new definition. Br Dent J. 2001;190(8):424-8. DOI: 10.1038/ sj.bdj.4800992a.

23. Cameron CE. Cracked-tooth syndrome. J Am Dent Assoc. 1964;68:405-11

24. Rosen H. Cracked tooth syndrome. J Prosthet Dent. 1982;47(1):36-43.

25. Wiebusch FB. Hairline fracture of a cusp: report of case. $J$ Can Dent Assoc (Tor). 1972;38(5):192-4.

26. Račić A, Dimitrijević M, Dukić V. The most often causes of odontogenic maxillary sinusitis. Vojnosanit Pregl. 2004;61(6):645-8

27. Račić A, Dotlić J, Janošević L. Oral surgery as risk factor of odontogenic maxillary sinusitis. Srp Arh Celok Lek. 2006;134(5-6):191-4. 
28. Brook I. Sinusitis of odontogenic origin. Otolaryngol Head Neck Surg. 2006;135(3):349-55. DOI: 10.1016/j.otohns.2005.10.059.

29. Cantín López M, Coronado Gallardo C, Suazo Galdames I, San Pedro Valenzuela J. Maxillary sinusitis of dental origin. A case report and literature review. Int J Odontostomat. 2009;3(1):5-9.

30. Mehra P, Jeong D. Maxillary sinusitis of odontogenic origin. Curr Allergy Asthma Rep. 2009;9(3):238-43.

31. Longhini $A B$, Ferguson BJ. Clinical aspects of odontogenic maxillary sinusitis: a case series. Int Forum Allergy Rhinol. 2011;1(5):409-15. DOI: 10.1002/alr.20058.

32. Hamory BH, Sande MA, Sydnor A, Seale DL, Gwaltney JM. Etiology and antimicrobial therapy of acute maxillary sinusitis. J Infect Dis. 1979;139(2):197-202. DOI: 10.1093/ infdis/139.2.197.

33. Esparza Gómez G, López-Argüello Illana C, García Núñez JA Moreno López LA. Recurrent aphthous stomatitis: review and up-to-date. Medicina oral. 1998;3(1):18-35.

34. Akintoye SO, Greenberg MS. Recurrent aphthous stomatitis. Dent Clin North Am. 2005;49(1):31-47, vii-viii. DOI: 10.1016/j.cden.2004.08.001.

35. Scully C, Gorsky M, Lozada-Nur F. The diagnosis and management of recurrent aphthous stomatitis: a consensus approach. J Am Dent Assoc. 2003;134(2):200-7.

36. Milián-Masanet M, Sanchis-Bielsa JM. Penfigoides: Revisión y puesta al día. RCOE. 2004;9(4):429-34.

37. Al-Hashimi I, Schifter M, Lockhart PB, Wray D, Brennan M, Migliorati CA, et al. Oral lichen planus and oral lichenoid lesions: diagnostic and therapeutic considerations. Oral Surg Oral Med Oral Pathol Oral Radiol Endod. 2007;103(S25):e1-12. DOI: 10.1016/j.tripleo.2006.11.001.

38. López-Jornet P, Bermejo-Fenoll A. Treatment of pemphigus and pemphigoids. Med Oral Patol Oral Cir Bucal. 2005;10(5):410-1.

39. Sanadi RM, Chelani LR, Bhakkand SR, Sheth JK. Role of trauma from occlusion in periodontal disease. A controversy. IOSR-JDMS. 2016;15(9):118-22.

40. Rowland RW. Necrotizing ulcerative gingivitis. Ann Periodontol. 1999;4(1):65-73; discussion 78 .

41. Herrera D, Retamal-Valdes B, Alonso B, Feres M. Acute periodontal lesions (periodontal abscesses and necrotizing periodontal diseases) and endo-periodontal lesions. J Clin Periodontol. 2018;45(S20):S78-94. DOI: 10.1111/jcpe.12941.

42. Novak MJ. Necrotizing ulcerative periodontitis. Ann Periodontol. 1999;4(1):74-8. DOI: 10.1902/annals. 1999.4.1.74.

43. Malek R, Gharibi A, Khlil N, Kissa J. Necrotizing Ulcerative Gingivitis. Contemp Clin Dent. 2017;8(3):496-500. DOI: 10.4103/ccd.ccd_1181_16.

44. Abrams AM, Melrose RJ, Howell FV. Necrotizing sialometaplasia. A disease simulating malignancy. Cancer. 1973;32(1):1305. DOI: 10.1002/1097-0142(197307)32:1<130::aidcncr2820320118>3.0.co;2-8.

45. Brannon RB, Fowler CB, Hartman KS. Necrotizing sialometaplasia: a clinicopathologic study of sixty-nine cases and review of the literature. Oral Surg Oral Med Oral Pathol. 1991;72(3):317-25.

46. Bascones-Martínez A, Muñoz-Corcuera M, Cerero-Lapiedra R, Bascones-llundáin J, Esparza-Gómez G. Case report of necrotizing sialometaplasia. Med Oral Patol Oral Cir Bucal. 2011;16(6):e700-3. DOI: 10.4317/medoral.16789.

47. Imbery TA, Edwards PA. Necrotizing sialometaplasia: literature review and cas reports. J Am Dent Assoc. 1996;127(7):1087-892.
48. El Deeb M, Holte N, Gorlin RJ. Submandibular salivary gland sialoliths perforated through the oral floor. Oral Surg Oral Med Oral Pathol. 1981;51(2):134-9.

49. Harrison JD, Epivatianos A, Bhatia SN. Role of microliths in the aetiology of chronic submandibular sialadenitis: a clinicopathological investigation of 154 cases. Histopathology. 1997;31(3):237-51

50. Rebolledo Cobos M, Carbonell Muñoz Z, Díaz Caballero A. Sialoliths in ducts and salivary glands. Literature review. Av Odontoestomatol. 2009;25(6):311-7.

51. Dulguerov P, Marchal F, Lehmann W. Postparotidectomy facial nerve paralysis: possible etiologic factors and results with routine facial nerve monitoring. Laryngoscope. 1999:109(5): 754-62.

52. Capaccio P, Ottaviani F, Manzo R, Schindler A, Cesana B. Extracorporeal lithotripsy for salivary calculi: a long-term clinical experience. Laryngoscope. 2004;114(6):1069-73. DOI: 10.1097/00005537-200406000-00021

53. Ospina AM, del Valle AF, Naranjo RF. Inflamación de glándulas salivales, revisión bibliográfica. Rev Fac Odon Univ Ant 2003;15(2):17-28.

54. Rauch S, Gorlin RJ. Diseases of the salivary glands. In: Gorlin RJ, Goldmann HM, eds. Thomas' Oral Pathology (6th ed.). St Louis: Mosby; 1974. p. 997-1003.

55. Rabinov JD. Imaging of salivary gland pathology. Radiol Clin North Am. 2000;38(5):1047-57.

56. Ruiz Veguilla E, Barrios Recio A, Díaz Caparros F. Patología no tumoral de las glándulas salivales. In: Libro virtual de formación en otorrinolaringología. Sociedad Española de Otorrinolaringología y Patología Cervico-Facial (SEORL-PCF); 2014: cap. 147 (1-9).

57. Arduino PG, Porter SR. Oral and perioral herpes simplex virus type 1 (HSV-1) infection: review of its management. Oral Dis. 2006;12(3):254-70. DOI: 10.1111/j.1601$0825.2006 .01202 . x$

58. Quindós G. Nuevas perspectivas en la terapia antifúngica. Gac Med Bilbao 2001;98:20-3.

59. Aguirre Urizar JM. Oral candidiasis. Rev Iberoam Micol. 2002;19(1):17-21.

60. Lopez-Jornet P, Molino Pagan D, Andujar Mateos P, Rodriguez Agudo C, Pons-Fuster A. Circadian rhythms variation of pain in burning mouth syndrome. Geriatr Gerontol Int. 2015;15(4):490-5. DOl: 10.1111/ggi.12303.

61. Barker KE, Savage NW. Burning mouth syndrome: an update on recent findings. Aust Dent J. 2005;50(4):220-3.

62. Maltsman-Tseikhin A, Moricca P, Niv D. Burning mouth syndrome: will better understanding yield better management? Pain Pract. 2007;7(2):151-62. DOI: 10.1111/j.15332500.2007.00124.x

63. Fedele S, Fricchione G, Porter SR, Mignogna MD. Burning mouth syndrome (stomatodynia). QJM. 2007;100(8):52730. DOl: 10.1093/qjmed/hcm049.

64. Klasser GD, Fischer DJ, Epstein JB. Burning mouth syndrome: recognition, understanding, and management. Oral Maxillofac Surg Clin North Am. 2008;20(2):255-71, vii. DOI: 10.1016/j.coms.2007.12.012

65. López-Jornet P, Camacho-Alonso F, Andujar-Mateos P. A prospective randomized study on the efficazy of tongue protector in patients with burning mouth sindrome. Oral dis. 2011:17(3):277-82

66. Rodríguez-de Rivera-Campillo E, López-López J. Evaluation of the response to treatment and clinical evolution in patients with burning mouth syndrome. Med Oral Patol Oral Cir Bucal. 2013;18(3):e403-10. DOI: 10.4317/medoral.18142. 
67. Algozaín Acosta Y, Viñas García M, Capote Leyva E, Rodríguez Llanes R. Clinical behavior of the dysfunction pain temporomandibular joint syndrome assessed in a Stomatology emergence consultation. Rev Cubana Estomatol. 2009;46(2): 7-8.

68. Sarlani E, Balciunas BA, Grace EG. Assessment and management of musculoskeletal and neuropathic causes. AACN Clin Issues. 2005;16(3):333-46.

69. Dimitroulis G. Temporomandibular disorders: A clinical update. BMJ. 1998;317(7152):190-4. DOI: 10.1136/ bmj.317.7152.190.

70. Giamberardino MA, Affaitati G, Fabrizio A, Constantini R Myofascial pain syndromes and their evaluation. Best Pract Res Clin Rheumatol. 2011;25(2):185-98. DOI: 10.1016/j. berh.2011.01.002.

71. Villaseñor Moreno JC, Escobar Reyes VH, de la Lanza Andrade LP, Guizar Ramírez BI. Síndrome del dolor miofascial. Epidemiología, fisiopatología, diagnóstico y tratamiento. Rev Esp Med Quir. 2013;18(2):148-57.

72. Ruiz M, Nadador V, Fernández-Alcantud J, Hernández-Salván J, Riquelme I, Benito G. Dolor de origen muscular: dolor miofascial y fibromialgia. Rev Soc Esp Dol. 2007;14(1):36-44.

73. Siccoli MM, Bassetti CL, Sándor PS. Facial pain: clinical differential diagnosis. Lancet Neurol. 2006;5(3):257-67. DOI: 10.1016/S1474-4422(06)70375-1

74. Benoliel R, Gaul C. Persistent idiopathic facial pain. Cephalalgia. 2017;37(7):680-91. DOI: 10.1177/0333102417706349.

75. Headache Classification Subcommittee of the International Headache Society. The International Classification of Headache Disorders (2nd ed.). Cephalalgia. 2004;24(S1):9-160.

76. Rozen TD. Trigeminal neuralgia and glossopharyngeal neuralgia. Neurol Clin. 2004;22(1):185-206. DOI: 10.1016/ S0733-8619(03)00094-X.

77. MacDonald BK, Cockerell OC, Sander JW, Shorvon SD. The incidence and lifetime prevalence of neurological disorders in a prospective community-based study in the UK. Brain. 2000;123(Pt 4):665-76. DOl: 10.1093/brain/123.4.665.

78. Mueller D, Obermann M, Yoon MS, Poitz F, Hansen $\mathrm{N}$, Slomke MA, et al. Prevalence of trigeminal neuralgia and persistent idiopathic facial pain: a populationbased study. Cephalalgia. 2011;31(15):1542-8. DOI: 10.1177/0333102411424619.

79. Cruccu G, Gronseth G, Alksne J, Argoff C, Brainin M, Burchiel $\mathrm{K}$, et al. AAN-EFNS guidelines on trigeminal neuralgia management. Eur J Neurol. 2008;15(10):1013-28. DOl: 10.1111/j.1468-1331.2008.02185.x.

80. Straus DC, Ko AL, Sekhar LN. Trigeminal Neuralgia. In: Ellenbogen RG, Sekhar LN, Kitchen N, eds. Principles of neurological surgery (4th ed.). China: Elsevier Inc; 2018. p. 745-52.

81. Baad-Hansen L. Atypical odontalgia - pathophysiology and clinical management. J Oral Rehabil. 2008;35(1):1-11. DOl: 10.1111/j.1365-2842.2007.01813.x.

82. Headache Classification Committee of the International Headache Society. Classification and diagnostic criteria for headache disorders, cranial neuralgias and facial pain. Cephalalgia. 1988;8(S7): 1-96.

83. Woda A, Pionchon P. A unified concept of idiopathic orofacial pain: clinical features. J Orofac Pain. 1999;13(3):172-84; discussion 185-95.

84. Lascelles RG. Atypical facial pain and depression. Br J Psychiatry. 1966;112(488):651-9.

85. Gross SG. Atypical odontalgia: a cause for dental failure. J Conn State Dent Assoc. 1991;67(2):36-7.
86. Pertes RA, Bailey DR, Milone AS. Atypical odontalgia - a nondental toothache. J N J Dent Assoc. 1995;66(1):29-33.

87. Lilly JP, Law AS. Atypical odontalgia misdiagnosed as odontogenic pain: a case report and discussion of treatment. J Endod. 1997;23(5):337-9. DOl: 10.1016/S0099-2399(97)80419-0.

88. Marbach JJ, Raphael KG. Phantom tooth pain: a new look at an old dilemma. Pain Med. 2000;1(1):68-77. DOI: 10.1046/j. 1526-4637.2000.00012.x.

89. Scully C, Bagan J. Oral squamous cell carcinoma overview. Oral Oncol. 2009;45(4):301-8. DOl: 10.1016/j.oraloncology.2009.01.004.

90. Johnson NW, Jayasekara P, Amarasinghe AA. Squamous cell carcinoma and precursor lesions of the oral cavity: Epidemiology and aetiology. Periodontol 2000. 2011;57(1):19-37. DOl: 10.1111/j.1600-0757.2011.00401.x

91. da Silva SD, Ferlito A, Takes RP, Brakenhoff RH, Valentin MD, Woolgar JA, et al. Advances and applications of oral cancer basic research. Oral Oncol. 2011;47(9):783-91. DOI: 10.1016/j. oraloncology.2011.07.004.

92. Friedrich RE. Delay in diagnosis and referral patterns of 646 patients with oral and maxillofacial cancer: a report from a single institution in Hamburg, Germany. Anticancer Res. 2010;30:1833-6

93. Kim KY, Lee GY, Cha IH. Biomarker detection for the diagnosis of lymph node metastasis from oral squamous cell carcinoma. Oral Oncol. 2012;48(4):311-9. DOI: 10.1016/j. oraloncology.2011.11.010.

94. Stefanuto P, Doucet JC, Robertson C. Delays in treatment of oral cancer: a review of the current literature. Oral Surg Oral Med Oral Pathol Oral Radiol. 2014;117(4):424-9. DOI: 10.1016/j.0000.2013.12.407.

95. Stokman MA, Spijkervet FK, Boezen HM, Schouten JP, Roodenburg JL, de Vries EG. Preventive intervention possibilities in radiotherapy- and chemotherapy-induced oral mucositis: results of meta-analyses. J Dent Res. 2006;85(8):690-700.

96. Kubota K, Kobayashi W, Sakaki H, Nakagawa H, Kon T, Mimura M, et al. Professional oral health care reduces oral mucositis pain in patients treated by superselective intraarterial chemotherapy concurrent with radiotherapy for oral cancer. Support Care Cancer. 2015;23(11):3323-9. DOI: 10.1007/s00520-015-2774-x.

97. Bendtsen L, Jensen R. Tension-type headache: the most common, but also the most neglected headache disorder. Curr Opin Neurol. 2006;19(3):305-9. DOI: 10.1097/01. wco.0000227043.00824.a9.

98. Aczél T, Kun J, Szöke É, Rauch T, Junttila S, Gyenesei A, et al. Transcriptional alterations in the trigeminal ganglia, nucleus and peripheral blood mononuclear cells in a rat orofacial pain model. Front Mol Neursci. 2018;11:219.

99. Stovner LJ, Hagen K, Jensen R, Katsarava Z, Lipton RB, Scher Al, et al. The global burden of headache: a documentation of headache prevalence and disability worldwide. Cephalalgia. 2007;27:193-210. DOI: 10.1111/j.14682982.2007.01288.x.

100. Jensen R. Peripheral and central mechanisms in tensiontype headache: an update. Cephalalgia. 2003;23(51):49-52. DOI: 10.1046/j.1468-2982.2003.00574.x

101. Lipton RB, Newman LC. Epidemiology, impact, and comorbidities of migraine headaches in the United States. Neurology. 2003;60(7):S3-8.

102. Schürks M, Kurth T, de Jesus J, Jonjic M, Rosskopf D, Diener HC. Cluster headache: clinical presentation, lifestyle features, and medical treatment. Headache. 2006;46(8):1246-54. DOI: 10.1111/j.1526-4610.2006.00534.x. 
103. Diener HC, Pfaffenrath V, Pageler L, Peil H, Aicher B. The fixed combination of acetylsalicylic acid, paracetamol and caffeine is more effective than single substances and dual combination for the treatment of headache: a multicentre, randomized, double-blind, single-dose, placebo-controlled parallel group study. Cephalalgia. 2005;25(10):776-87. DOI: 10.1111/j.1468-2982.2005.00948.x.

104. Hanson MB, Glasscock ME 3rd, Brandes JL, Jackson CG. Medical treatment of headache after suboccipital acoustic tumor removal. Laryngoscope. 1998;108(8 Pt 1):1111-4. 